\title{
The Genetic Control of Two Lethal Traits in Variety 1, Tetrahymena pyriformis*
}

EDUARDO ORIAS $+\ddagger$

Department of Zoology, University of Michigan, Ann Arbor, Michigan

SYNOPSIS. A breeding analysis of a wild strain (Indian Lake 12) of variety 1, Tetrahymena pyriformis, discloses recessive lethal alleles at two independently assorting loci. Conclusions concerning the cytogenetic behavior of this species have been verified through the scoring of a large number of offspring

$\mathrm{T}$ HE FIRST demonstration of Mendelian behavior in variety 1 of Tetrahymena pyriformis came through the analysis of mating types (7). Because the genotypes in this case could be ascertained only through extensive progeny tests, the number of segregants which could be studied was limited, and the possibility of rare cytogenetic anomalies, such as cytogamy, could not be excluded. The present study establishes genetic control for two lethal traits. Furthermore, since the genotypes of clones are expressed directly in the phenotype, large samples of progeny can be examined and a critical search for certain cytogenetic aberrations is possible.

\section{MATERIALS AND METHODS}

Biological materials. Two strains of variety $1, T$. pyriformis, were used for the study. One, Indian Lake 12 (IL-12), was collected by Nanney at Indian Lake State Park, Michigan. The other was an inbred strain belonging to family $A(7)$. The latter clone was past the "inbreeding crisis" (cf. 3) and is presumably homozygous for most of its loci. All of its lethal and deleterious alleles have been removed by laboratory selection, and essentially $100 \%$ viability of the progeny is obtained at every subsequent inbreeding step.

Description of the lethal traits. Many offspring in backcrosses of F1 hybrids to the IL-12 strain were inviable. Those pair isolates which failed to become established following conjugation underwent a distinctive sequence of changes which led to the death of the offspring. The exconjugants, normally reduced in size after conjugation, failed to grow and became progressively smaller until, after 3-4 days, they could no longer be found under the dissecting microscope. Cells

* This work was partially supported by a grant from the National Science Foundation to Dr. D. L. Nanney.

† Present address: Department of Biological Sciences, Santa

Barbara College, University of California, Goleta, California.

$\ddagger$ The author wishes to express his gratitude to Dr. David L Nanney for his encouragement, advice and criticism during all stages of this work, and to Dr. Ray D. Owen for his critical reading of the manuscript. segregating the lethals. In particular, cytogamy does not occur in these strains with a frequency higher than $2 \%$, if indeed it occurs at all. The finding of Iethal heterozygosity in a wild strain suggests that close inbreeding usually does not take place in nature and supports the conclusion that variety 1 of $T$. pyriformis is an outbreeding species.

showing this lethal trait have been given the name "tiny."

During the genetic study of the "tiny" trait, another lethal trait clearly distinguishable from "tiny" was uncovered. Cells showing this trait, designated as "fat," resumed growth normally after separation of the exconjugants, but never underwent the first division following conjugation. Instead, they became approximately spherical and cytolysed 2-3 days later.

A third kind of lethality was also found. Infrequently, the exconjugants would become very large and/or lose their smooth shape. Invariably, in this case, at least one of the exconjugants was able to divide a few fissions before dying; some resumed normal growth. The low frequency and the variability of these anomalies implicate a complex mode of control, and such clones were excluded from consideration; they were scored as "normal" for the purposes of this study.

Cytogenetics. Detailed cytogenetic studies in variety 1 have been carried out previously (2.8-10). The diploid number of chromosomes is 10 ( 5 pairs). The relevant events of conjugation are the following. The micronucleus in each pair member divides twice, meiotically, giving rise to 4 haploid nuclear products; of these, 3 disintegrate and one divides once more, mitotically, producing a migratory and a stationary pronucleus. The 2 pair members exchange migratory pronuclei and these fuse with the stationary pronuclei of the mates. The diploid fusion nucleus, or synkaryon, of each pair member divides twice. mitotically. giving rise to 4 diploid nuclear products: 2 polyploidize and become macronuclei, one remains as the diploid micronucleus and the other disintegrates. At the first cell division following separation of the exconjugants the two macronuclei are passively distributed, one to each daughter cell, while the micronucleus divides mitotically. The result of the process described is the genotypic identity of the two fertilization nuclei and. consequently, of the sister exconjugants. Thus, the 2 exconjugants of a pair are expected to be alike for 
any hereditary trait directly controlled by the genotype.

Culturing techniques. The techniques used have been described earlier $(6,7)$. Cultures were maintained in a $0.15 \%$ infusion of rye "Cerophyl" (Cerophyl Laboratories, Inc.) inoculated the previous day with a pure culture of Aerobacter aerogenes. Stocks were maintained in test tubes with $5 \mathrm{cc}$. of culture medium. The cultures were kept at $15^{\circ} \mathrm{C}$., and were fed every 6 months by replacing half of the volume with fresh culture fluid. Clones were grown in 3-spot depression slides filled with $0.6 \mathrm{cc}$. of cuiture medium, at room temperature.

Crosses. Stocks maintained at $15^{\circ} \mathrm{C}$. were prepared for crossing by transferring to room temperature and feeding once, $2-3$ days in advance. At the time of crossing, the two clones were fed once more, and mixed in the middle depression of a 3-spot depression slide. Each outside depression was filled with one of the two unmixed clones as controls. At this time the slides were placed in an incubator kept at $26^{\circ} \mathrm{C}$. In the evening of the next day single pairs were isolated into depressions containing a single drop of cultured medium. By early the following morning the pair members had separated; one member of each pair was transferred to a single drop of culture medium in a new depression, while the other member was left undisturbed in the old one. After allowing one day for the exconjugants to start dividing, the depressions were scored for the presence of "tiny" and "fat" cells; depressions containing more than one cell were filled with $0.6 \mathrm{cc}$. of culture medium, while the rest were left undisturbed. Two days later-3 days after separation of the exconjugants-those depressions still containing a single drop were checked again, while from each of the remainder 1-20 cells were transferred to a second depression. After checking the 1 st and 2nd depression cultures for non-conjugation (see below) they were discarded.

The original cross of IL-12 to the inbred strain constitutes the only exception to the terminal portion of the procedure outlined above. In this case, serial single cell transfers of the cultures were made every 2 days. The 7 th depression culture was transferred to a test tube containing $3 \mathrm{cc}$. of medium and tested for mating type according to the usual procedure.

Test for non-conjugation. Cells may unite and remain together for a considerable period of time, but separate without completing meiosis, fertilization and macronuclear development. Once a clone from a pair is established, it therefore becomes essential to ascertain whether the clone is the product of a true sexual union, or whether it represents a vegetative descendant of one of the parental cells. The most efficient technique devised for the detection of "non-conjugant" clones (7), makes use of the fact that when a pair undergoes true sexual union, the exconjugants and their vegetative descendants usually become sexually immature-i.e. incapable of mating-and remain so for a period of 50-80 cell divisions. If the sexual union is not consummated, on the other hand, no such immaturity will be detected. Therefore, cases of nonconjugation were excluded by testing the 1 st and 2 nd depression cultures of both exconjugants of every pair. The tests were performed by mixing each culture with a tester differing in mating type from either parental clone, and scoring for the presence or absence of mating on the next day. Under the conditions of this study, non-conjugants occurred with a frequency of less than $3 \%$.

Breeding scheme. The wild strain IL-12 was crossed to an inbred strain of family $\mathrm{A}(7)$ yielding a $100 \%$ viable $F 1$ generation. The two exconjugants from each of $44 \mathrm{~F} 1$ pairs were raised to maturity and their mating types determined. In order to be certain that all the $\mathrm{F} 1$ clones used in progeny tests were true conjugants, the 8 sets of exconjugants expressing the parental combination of mating types, I and III. were excluded. The sister exconjugants of $25 \mathrm{~F} 1$ pairs were crossed inter se, and one member of each set was backcrossed to the IL-12 parental strain. This procedure could not be carried out with the descendants of the remaining $11 \mathrm{~F} 1$ pairs because, in each case. both exconjugants expressed the same mating type as IL-12. They were, therefore, also excluded. Multiples of 15 pairs were isolated in each cross.

\section{RESULTS AND CONCLUSIONS}

In the first crosses the exconjugants from each pair were isolated soon after they had separated. The pairs isolated fell in one of 3 classes: those that yielded 2 normal exconjugants, those that yielded 2 "tiny" exconjugants, and those that yielded 2 "fat" exconjugants. Sine the genotypes of exconjugants are identical, the perfect phenotypic correlation between the 2 exconjugants immediately suggested a direct genetic control of these traits.

In later crosses, the two exconjugants were occasionally permitted to remain together, but whenever a "tiny" exconjugant was detected in a depression, a careful search revealed the presence of the other "tiny" exconjugant; similarly, whenever a "fat" exconjugant was detected, another "fat" exconjugant was invariably found. Thus it is certain that a "tiny" "fat" combination of sister exconjugants never arose in this study. The normal-"fat" and normal-"tiny" combinations were never observed, but this absence is not as critical.

The results of backcrossing and inbreeding the Fl clones are shown in Table I. The following conclu- 
TABLE I. The frequencies of normal, "tiny" and "fat" offspring obtained in the backcrosses and inbreeding of the Fl progeny of wild strain IL-12.

\begin{tabular}{|c|c|c|c|c|c|c|c|c|c|}
\hline $\begin{array}{l}\text { Class and } \\
\text { proposed } \\
\text { genotype }\end{array}$ & $\begin{array}{l}\text { F1 clone } \\
\text { number }\end{array}$ & $\begin{array}{c}\text { Backc } \\
\text { normal }\end{array}$ & $\begin{array}{l}\text { ross to } \\
\text { "fat" }\end{array}$ & $\begin{array}{l}\text { IL-12 (F } \\
\text { "tiny" }\end{array}$ & $\begin{array}{l}\text { fTt) } \\
\text { total* }\end{array}$ & $\begin{array}{l}\text { Inbreedi } \\
\text { normal }\end{array}$ & $\begin{array}{l}\text { ng of sis } \\
\text { "fat" }\end{array}$ & $\begin{array}{l}\text { ster excor } \\
\text { "tiny" }\end{array}$ & $\begin{array}{l}\text { jugants } \\
\text { total* }\end{array}$ \\
\hline \multirow{10}{*}{$\underset{(\mathrm{FFTT})}{\mathrm{A}}$} & 3 & 30 & - & - & 30 & \multirow{4}{*}{114} & \multirow{4}{*}{-} & \multirow{4}{*}{-} & \multirow{4}{*}{114} \\
\hline & 4 & 29 & - & - & 29 & & & & \\
\hline & 5 & 29 & - & - & 29 & & & & \\
\hline & 2.3 & .30 & $\ldots$ & - & 30 & & & & \\
\hline & 29 & 45 & 一 & - & 45 & \multirow{4}{*}{$\begin{array}{l}30 \\
42\end{array}$} & \multirow{4}{*}{-} & \multirow{4}{*}{ - } & \multirow{4}{*}{$\begin{array}{l}30 \\
42\end{array}$} \\
\hline & 31 & 45 & - & - & 45 & & & & \\
\hline & 39 & 29 & - & - & 29 & & & & \\
\hline & 60 & 30 & - & - & 30 & & & & \\
\hline & Sub-Total & 267 & - & - & 267 & 186 & - & - & 186 \\
\hline & Total & & & & & 453 & - & - & 453 \\
\hline \multirow{10}{*}{$\begin{array}{c}\mathrm{B} \\
(\mathrm{FFTt})\end{array}$} & 13 & 25 & - & 5 & 30 & 20 & - & 10 & 30 \\
\hline & 22 & 25 & - & 5 & 30 & 26 & - & 4 & 30 \\
\hline & 25 & 22 & - & 5 & 27 & 22 & - & 8 & 30 \\
\hline & 26 & 22 & - & 6 & 28 & 20 & - & 9 & 29 \\
\hline & 30 & 18 & -- & 9 & 27 & & & & \\
\hline & 47 & 23 & - & 7 & 30 & 27 & -. & 3 & 30 \\
\hline & 48 & 21 & - & 8 & 29 & 22 & - & 8 & 30 \\
\hline & 52 & 27 & -- & 11 & 38 & 47 & - & 13 & 60 \\
\hline & Sub-Total & \multirow[t]{2}{*}{183} & \multirow[t]{2}{*}{-} & \multirow[t]{2}{*}{56} & \multirow[t]{2}{*}{239} & 184 & - & 55 & 239 \\
\hline & Total & & & & & 367 & - & 111 & 478 \\
\hline \multirow{5}{*}{$\begin{array}{c}\mathrm{C} \\
(\mathrm{FfTT})\end{array}$} & 9 & 24 & 6 & - & 30 & 20 & 6 & - & 26 \\
\hline & 17 & 16 & 10 & - & 26 & 48 & 12 & $\ldots$ & 60 \\
\hline & 33 & 19 & 9 & - & 28 & 20 & 10 & - & 30 \\
\hline & Sub-Total & 59 & 25 & - & 84 & 88 & 28 & - & 116 \\
\hline & Total & & & & & 147 & 53 & - & 200 \\
\hline \multirow{8}{*}{$\underset{(\mathrm{FfTt})}{\mathrm{D}}$} & 10 & 18 & 3 & 9 & 30 & \multirow{3}{*}{30} & \multirow{3}{*}{15} & \multirow{3}{*}{14} & \multirow{3}{*}{59} \\
\hline & 14 & 12 & 10 & 7 & 29 & & & & \\
\hline & 22 & 18 & 4 & 8 & 30 & & & & \\
\hline & 28 & 13 & 7 & 10 & 30 & 50 & 20 & 19 & 89 \\
\hline & 45 & 17 & 6 & 7 & 30 & 14 & 7 & 9 & 30 \\
\hline & 57 & 17 & 4 & 9 & 30 & 50 & 19 & 21 & 90 \\
\hline & Sub-Total & 95 & 34 & 50 & 179 & 144 & 61 & 63 & 268 \\
\hline & Total & & & & & 239 & 95 & 113 & 447 \\
\hline
\end{tabular}

* The difference between the total off spring scored in a cross and the next higher multiple of 15 represents clones excluded by tests for non-conjugation (see text).

sions can be derived from this table:

1) The backcrosses and the corresponding F2 crosses of sister exconjugants fall in four classes: those in which "tiny" cells segregate (Class B), those in which "fat" cells segregate (Class C), those in which both segregate (Class D) and those in which neither segregates (Class A).

2) If the progeny of those crosses in which "tiny" cells segregate ( $\mathrm{B}$ and $\mathrm{D}$ ) are pooled, the resulting ratio of non-"tiny" to "tiny" cells is 701:224, not significantly different from a $3: 1$ ratio $(\mathbf{P}=0.6-0.5)$. The ratio of crosses segregating "tiny" $(\mathrm{B}+\mathrm{D})$ to those not segregating them $(\mathrm{A}+\mathrm{C})$ is $14: 11$, not significantly different from a $1: 1$ ratio $(P=0.6-0.5)$.

3 ) If the progeny of crosses in which "fat" cells segregate $(\mathrm{C}$ and $\mathrm{D})$ are pooled, the resulting ratio of normal to "fat" cells is $386: 148$, not significantly different from a 3.1 ratio $(P=0.2-0.1)$. The ratio of crosses segregating "fat" $(\mathrm{C}+\mathrm{D})$ to those not segregating them is $9: 16$, not significantly different from a $1: 1$ ratio $(P=0.2-0.1)$. It has been assumed that a cell expressing the "tiny" phenotype cannot be scored with respect to the "fat" trait; consequently, "tiny" cells produced in crosses of Class D have been excluded from the computations just described. The validity of this assumption is justified below.

The data are thus consistent with the hypothesis that the lethal "tiny" phenotype is controlled by a recessive allele of a locus designated as the "tiny" or $\mathrm{T}$ locus, and the "fat" phenotype is controlled by a recessive allele of another locus, designated as the "fat" or F locus. The IL-12 strain is therefore assumed to be heterozygous for both loci.

The interaction between these two loci in transmission (linkage) and in expression (epistasis) can be analyzed by reference to the di-hybrid crosses (Class D). The ratios of the three types of offspring in the backcrosses are not significantly different from the ratios in the corresponding inbreeding ( $P=0.5-0.4)$ and the data from the 2 kinds of crosses can be pooled. The ratio thus obtained, 239:95:113 normal:"fat": "tiny" is not significantly different from a 9:3:4 ratio 
$(\mathrm{P}=0.4-0.3)$, but it does differ highly significantly from either a $9: 4: 3$ or a $9: 3: 3: 1$ ratio $(\mathrm{P}<0.01)$. The ratios among the progeny are thus consistent with the assumptions that the $\mathrm{T}$ locus is epistatic over the F locus-i.e., cells of the genotypes FFtt, Fft and fftt are phenotypically indistinguishable under the conditions used in the present study-and that these two loci assort independently. However, since linkage calculations from di-hybrid crosses are less stringent than those from testcrosses, linkage with a crossover frequency as high as $45 \%$ would not be statistically incompatible with these data. The $8: 8: 3: 6$ ratio of $A: B: C: D$ classes in the $F 1$ is not significantly different from a $1: 1: 1: 1$ ratio $(P=0.7-0.6)$ and agrees with the assumption of random assortment of the two loci. This hypothesis concerning the genetic control of the two traits leads to the following predictions:

1) Since the inbred parental strain (family A) is doubly homozygous for the normal alleles (FFTT), the backcross of any F1 clone to that parent should yield only normal offspring. Single clones representing each of classes B, C and D of F1 clones were backcrossed to this parent, and the 30 pairs isolated in each case gave rise to normal offspring.

2) When a double heterozygote, FfTt, is crossed to either of the single heterozygotes, FfTT or FFTt, the one, but not both, lethals should segregate in each cross. A cross between $F 1$ clones of classes $C$ and $D$ (FfTT $\times$ FfTt) yielded 26 normal and 3 "fat" sets of exconjugants, a ratio not significantly different from the $3: 1$ ratio expected $(\mathrm{P}=0.2-0.1)$. A cross between strains of classes $B$ and D (FFTt $\times$ FfTt) yielded 24 normal and 5 "tiny" sets of exconjugants, a ratio not significantly different from the $3: 1$ ratio expected $(\mathrm{P}=0.5-0.4)$.

3) When a single heterozygote is crossed to a clone singly heterozygous for the other locus (FfTT $X$ FFTt), all the progeny of the cross should be phenotypically normal. Thirty pairs isolated from a cross between clones of classes $\mathrm{B}$ and $\mathrm{C}$ yielded 30 normal offspring.

Thus all of the results are in agreement with the hypothesis that the lethal traits designated as "tiny" and "fat" are controlled by recessive alleles of two independently assorting loci.

The upper limit to the frequency of cytogamy. Cytogamy is a process, first described in Paramecium caudatum (14), whereby 2 paired cells will synchronously undergo self-fertilization, i.e., autogamy (cf. 11). The functional product of meiosis in each pair member gives rise, mitotically, to the migratory and stationary pronuclei; but the migratory pronuclei, instead of being exchanged, fuse with their sister stationary pronuclei. This process, while restoring the diploid condition of each "exconjugant," leads to com- plete homozygosis for every locus. The 2 "exconjugants," moreover, will not necessarily be identical in genotype, as expected for true exconjugants.

The internal consistency of the interpretation provided for the control of the two lethal traits depends on the assumption that cytogamy occurs very infrequently, if it occurs at all. Although no evidence of cytogamy has been detected, a statistical analysis of the data available provides an estimate of the upper limit to the frequency of cytogamy in laboratory crosses of the strains used.

Since cytogamy disturbs the perfect genotypic correlation between sister exconjugants, it should yield new combinations of sister exconjugant phenotypes. namely normal-"fat", normal-"tiny" and "tiny"-fat". It is possible that the first two combinations were always missed if they occurred, because in many of the later crosses the depressions were examined after the exconjugants had divided at least once. It is not possible to estimate accurately how frequently this may have happened and, consequently, it will be assumed that any pairs which yielded normal."tiny" and normal-"fat" combinations of exconjugants were always mis-scored as normal. This assumption excludes some of the information contained in the data and biases the estimate of the upper limit by making it too high.

While the lack of distortion of the $3: 1$ or $9: 3: 4$ ratios contributes some information to the lowering of the upper limit to the frequency of cytogamy, this information is negligible when compared to that furnished by the absence of the "tiny"-ffat" class of sister exconjugants. Again, neglecting the information from ratios overestimates slightly the upper limit.

Only 3 kinds of crosses from which large numbers of progeny are available could yield, through cytogamy, "tiny"-"fat" sets of exconjugants. namely FfTt $\times$ FfTt, FfTt $\times$ FFTt and FfTt $\times$ FfTT. As shown in Table II, $1 / 4$ of the pairs isolated in a cross of the 1st kind (FfTt $\times$ FfTt) should yield "tiny""fat" sets of exconjugants if all the pairs are cytogamous. Similarly, $1 / 8$ of the pairs isolated in crosses of the 2 nd kind (FfTt $\times$ FFTt), and $/ / 4$ of those isolated in crosses of the 3 rd kind (FfTt $\times$ FfTT), should yield "tiny"-"fat" sets of exconjugants if all the pairs are cytogamous. The total number of such pairs, expected among the 770 pairs isolated in crosses of the 3 kinds, is 163 (see Table III) if the frequency of cytogamy is 1 , i.e., $100 \%$. If that frequency is instead assumed to be $\mathrm{p}$-where $\mathrm{p}$ is a constant. independent of the kind of cross-the expected number of exceptional pairs becomes 163 p. By equating this number to 3.7 -the upper limit for a 0 observation, at the $5 \%$ level of significance (13)-and solving for $\mathrm{p}$, the upper limit to the frequency of cytogamy can be estimated to be $2.3 \%(3.7: 163)$. In view of the 
TABLE II. The frequencies of sister exconjugant combinations expected in a cross between clones of genotype Ff'Tt if all the pairs isolated were cvtogamous.

\begin{tabular}{|c|c|c|c|c|c|}
\hline \multirow{2}{*}{\multicolumn{2}{|c|}{$\begin{array}{l}\text { Exconjugant } \\
\text { Genotypes } \\
\text { Phenotypes } \\
\text { and their } \\
\text { Probability }\end{array}$}} & \multicolumn{4}{|c|}{ Second exconjugant } \\
\hline & & $\begin{array}{c}\text { FFTT } \\
\text { normal } \\
1 / 4\end{array}$ & $\begin{array}{c}\text { ffTT } \\
\text { "fat" } \\
1 / 4\end{array}$ & $\underset{\substack{\mathbf{1} / 4 \\
\text { "tiny" }}}{\mathbf{F F t t}}$ & $\begin{array}{c}\text { fftt } \\
\text { "tiny" } \\
1 / 4\end{array}$ \\
\hline \multirow{4}{*}{ 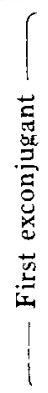 } & $\begin{array}{c}\text { FFTT } \\
\text { normal } \\
1 / 4\end{array}$ & $\begin{array}{c}\text { normal-normal } \\
1 / 16\end{array}$ & $\underset{1 / 16}{\text { normal-"fat"* }}$ & $\begin{array}{c}\text { normal-"tiny"* } \\
1 / 16\end{array}$ & $\begin{array}{c}\text { normal-"tiny" } \\
1 / 16\end{array}$ \\
\hline & $\begin{array}{c}\text { ff'TT } \\
\text { "fat" } \\
1 / 4\end{array}$ & $\begin{array}{c}\text { normal-"fat"s } \\
1 / 16\end{array}$ & $\begin{array}{c}\text { "fat"-"fat" } \\
1 / 16\end{array}$ & $\begin{array}{c}\text { "tiny"-"fat" } \\
1 / 16\end{array}$ & $\begin{array}{c}\text { "tiny"-"fat" } \\
1 / 16\end{array}$ \\
\hline & $\begin{array}{c}\text { FFtt } \\
\text { "tiny" } \\
1 / 4\end{array}$ & $\underset{1 / 16}{\operatorname{normal} \text {-"tiny"* }}$ & $\begin{array}{c}\text { "tiny"-"fat" } \\
1 / 16\end{array}$ & $\begin{array}{c}\text { "tiny"-_tiny" } \\
1 / 16\end{array}$ & $\begin{array}{c}\text { "tiny"-"tiny" } \\
1 / 16\end{array}$ \\
\hline & $\begin{array}{c}\text { fftt } \\
\text { "tiny" } \\
1 / 4\end{array}$ & $\underset{1 / 16}{\operatorname{normal}(\text { tany" }}$ & $\begin{array}{c}\text { "tiny"-"fat" } \\
1 / 16\end{array}$ & $\begin{array}{c}\text { "tiny"-_tiny" } \\
1 / 16\end{array}$ & $\begin{array}{c}\text { "tiny"-"tiny" } \\
1 / 16\end{array}$ \\
\hline
\end{tabular}

* Assumed to have been mis-scored as normal (see text).

Expected ratio of exconjugant sets: 7 normal : 1 "fat" : 4 "tiny" : 4 "tiny"-"fat".

simplifying assumptions which bias the estimate, it is likely that the frequency of cytogamy, if greater than 0 , does not exceed $2 \%$ in the strains used.

TABLE III. The number of pairs from the 3 kinds of crosses (see text) expected to yield "tiny"-"fat" sets of exconjugants if all pairs isolated were cytogamous.

\begin{tabular}{|c|c|c|c|}
\hline $\begin{array}{l}\text { Genotypes } \\
\text { crossed }\end{array}$ & $\begin{array}{c}\text { Frequency } \\
\text { of cytcgamous } \\
\text { "tiny"-"fat" sets } \\
\text { of exconjugants } \\
\text { expected }\end{array}$ & $\begin{array}{c}\text { Total number } \\
\text { of pairs } \\
\text { isolated } \\
\text { (see Table I) }\end{array}$ & $\begin{array}{c}\text { Total number } \\
\text { of cytogamous } \\
\text { "tiny"-"fat" sets } \\
\text { of exconjugants } \\
\text { expected }\end{array}$ \\
\hline FfTt $\times$ FfTt & $1 / 4 *$ & 447 & 112 \\
\hline FfTt $\times$ FFTt & $1 / 8 \dagger$ & 239 & 30 \\
\hline FfTt $\times$ FfTT & $1 / 4 \dagger$ & 84 & 21 \\
\hline Total & & 770 & 163 \\
\hline
\end{tabular}

* See Table II

† Computation nct shown, analogous to that of Table II.

\section{DISCUSSION}

The data presented provide evidence for the existence of two more loci for which allelic differences are known in variety 1 of $T$. pyriformis. The only other locus described in detail for this species is the mating type locus; alleles of the locus control different spectra of mating types which clones can express through the early differentiation of their macronuclear subnuclei $(1,5,7)$. Evidence for a locus controlling antigenic potentialities is also available(4).

The lethal nature of the traits described in the present study makes it impossible to maintain homozygous stocks of the mutant alleles, and considerably reduces their usefulness as chromosomal markers in future genetic studies. However the study of the mutants has proven useful in reinforcing the genetic evidence for the cytogenetic behavior of this species during conjugation (7). Thus, the scoring of large numbers of offspring has confirmed the evidence that the two exconjugants of a pair always have identical genotype. In particular, the present study leads to the conclusion that cytogamy, one mechanism that would disrupt the genotypic correlation between sister exconjugants, could not occur in the strains studied with a frequency exceeding $2 \%$ if, indeed, it occurs at all.

The finding of heterozygosis for lethal alleles of two different loci in a wild strain, moreover, suggests that close inbreeding does not ordinarily occur in nature. and supports the conclusion that this species relies on an outbreeding genetic economy (cf. 12).

No attempt has been made to characterize in any finer detail the nature of the defect which kills the homozygotes for either lethal allele; the material made available, however, might be useful for studies of developmental genetics. "Tiny" cells, for example, appear unable to resume growth following conjugation, and a study of the morphogenesis of the mutant cells might prove fruitful. "Fat" cells, on the other hand, appear to be blocked in their ability to undergo the first division following conjugation, and the nature of their defect thus appears to be quite different. The feasibility of growing these Tetrahymena cells in a chemically defined medium(2) provides an opportunity to explore the primary biochemical defects of the mutants, since specific supplementation of the medium might correct the lethal defects.

\section{REFERENCES}

1. Allen, S. L. \& Nanney, D. L. 1958. An analysis of nuclear differentiation in the selfers of Tetrahymena. Am. Naturalist 92, $139-60$.

2. Elliott, A. M. \& Hayes, R. E. 1953. Mating types in Tetrahymena. Biol. Bull. 105, 269-84.

3. Nanney, D. L. 1957. Inbreeding degeneration in Tetrahymena. Genetics 42, 137-46.

4. — 1959. Serotype determination in Tetrahymena pyriformis, variety 1. Genetics (in press). Abstract. 
5. Nanney, D. L. \& Allen, S. L. 1959. Intranuclear coordination in Tetrahymena. Physiol. Zool. (in press).

6: Nanney, D. L. \& Caughey, P. A. 1953. Mating type determination in Tetrahymena pyriformis. Proc. Natl. Acad. Sci. $39,1057-63$

7. Nanney, D. L., Caughey, P. A. \& Tefankjian, A. 1955. The genetic control of mating type potentialities in Tetrahymena pyriformis. Genetics 40, 668-80.

8. Ray, C. Jr. 1954. Chromosome behavior during conjugation of mating types I and II of variety 1 of Tetrahymena. Biol. Bull. 107, 318-19. Abstract.

9. - 1956. Meiosis and nuclear behavior in Tetrahymena pyriformis. J. Protozool. 3, 88-96.
10. Ray, C. Jr. \& Elliott, A. M. 1954. Chromosome number of four varieties of Tetrahymena pyriformis. Anat. Record 120, 812. Abstract.

11. Sonneborn, T. M. 1947. Recent advances in the genetics of Paramecium and Euplotes. Advances in Genetics 1, 263-358.

12. — 1957. Breeding systems, reproductive methods and species problems in the Protozoa, in Mayr, E., The Species Problem. AAAS, Washington, 155-324.

13. Stevens, W. L. 1942. Accuracy of mutation rates. $J$. Genetics 43, 301-7.

14. Wichterman, R. 1939. Cytogamy: a new sexual process in joined pairs of Paramecium caudatum. Nature 144, 123-4.

J. Protozool. 7(1), 69-74 (1960).

\title{
Volume, Nucleic Acid and Nitrogen Contents of Strains of Green and Colorless Euglena gracilis and of Astasia longa*
}

\author{
RU'TH H. NEFF
}

Department of Biology, Vanderbilt University, Nashville, Tennessee

SYNOPSIS. Nucleic acid, the nitrogen content per cell, and cell and nuclear volume were determined in 4 green and 2 heat-induced colorless strains of Euglena gracilis and one strain of Astasia longa.

All strains of Euglena were identical in cell and nuclear volume. The deoxyribonucleic acid content per cell of the apoplastidic strains was higher than that of the corresponding green strains by $1 \frac{1}{2}$ times. Although their nuclei were not enlarged, Feulgen staining of the colorless strains was also more

$\mathbf{M}^{\mathrm{A}}$ ANY permanently colorless (apoplastidic) strains of Euglena gracilis have been induced by treatment with streptomycin (SM) $(27,30)$, elevated temperatures (25), pyribenzamine (PBZ) (13), and ultraviolet irradiation (22).

The morphological consequences of apoplastidy have been fully described $(7,9,18,23,26,31,32)$. Although there are minor differences in sequence observed with the different agents, the authors cited agree that the chloroplasts are drastically reduced in size and number, altered in structure, and ultimately destroyed. The eyespot may be lost under certain conditions. There is often a simultaneous "hypertrophy" of mitochondria. The similarity in morphology of the apoplastidic strains of $E$. gracilis to the colorless flagellate Astasia longa has been noted $(23,24)$.

The loss of chloroplasts is accompanied by other changes: alteration of growth rate and total growth (12); reduced resistance to supra-optimal temperatures $(10,22)$; inhibition of chlorophyll synthesis $(2,7$, $12,15)$; disturbance of carotene and xanthophyll syntheses(8); and qualitative changes in the nucleotide ratios in ribonucleic acid(1).

* This investigation was supported by Research Grant No. E-1202 from the United States Public Health Service. Part of the data was presented at the 1957 AIBS meetings. intense. The significance of the increase in DNA in experimentally induced apoplastidy is discussed. As for $\mathrm{N}$ content, total nucleic acid $P$, and pentosenucleic acid-the differences between the strains reflected previously established morphological and physiological relationships between them.

The single strain of Astasia studied was identical in structure and size with the apoplastidic Euglena strains. However, it was quite unlike them in all the biochemical characteristics examined.

In this investigation, cell and nuclear volumes. total nucleic acid phosphorus, pentose nucleic acid (PNA), deoxypentose nucleic acid (DNA), and nitrogen contents of 7 strains of Euglena and Astasia were determined. The results demonstrate some additional biochemical consequences of experimentally (heat) induced apoplastidy.

\section{MATERIALS AND METHODS}

Six strains of $E$. gracilis and one of $A$. longa were analyzed:

1. E. gracilis var. bacillaris Pringsheim-University of Indiana Culture Collection, No. 160.

2. A. longa Pringsheim--University of Indiana, No. 626.

3. E. gracilis, Mainx strain-Pringsheim collection, 1224 5/A. (Hereafter abbreviated as Mx.)

4. E. gracilis, Stamm R (grüne)-Pringsheim Collection, No. $12245 / 13$. $\left(\mathrm{R}_{\mathrm{g}}\right)$.

5. E. gracilis, Stamm R (farblos)-Pringsheim Collection, No. $12245 / 13$. $\left(\mathrm{R}_{\mathrm{f}}\right)$.

6. E. gracilis, Stamm T (grüne)-Pringsheim Collection, $12245 / 9 .\left(\mathrm{T}_{\mathrm{g}}\right)$.

7. E. gracilis, Stamm T (farblos)-_Pringsheim Collection, $12245 / 9$. $\left(\mathrm{T}_{\mathrm{f}}\right)$.

The first two strains are described briefly in the 